\title{
Expression of a PYCARD/ASC variant lacking exon 2 in Japanese patients with palindromic rheumatism increases interleukin- $1 \beta$ secretion
}

\author{
Yumi Suganuma, ${ }_{1}$ Hayate Tanaka, ${ }^{1}$ Aya Kawase, ${ }^{1}$ Aoi Kishida,,${ }^{1}$ Moeko Yamaguchi,,${ }^{1}$ Atsuko Yabuuchi, ${ }^{1}$ Koji Inoue, ${ }^{2}$ \\ Shunichi Shiozawa, ${ }^{3}$ Koichiro Komai ${ }^{1 *}$
}

\begin{abstract}
Background: Palindromic rheumatism (PR) is a rare periodic arthritis characterized by relapsing short episodes of arthritis. Although the pathogenesis of PR is still unclear, the clinical condition is similar to that of autoinflammatory diseases caused by dysregulation of inflammasome-related genes.
\end{abstract}

Objective: We analyzed the inflammasome adapter PYD and CARD domain-containing protein/apoptosis-associated speck-like protein containing a CARD (PYCARD/ASC) in Japanese patients with PR.

Methods: Serum interleukin (IL)-1 $\beta$ concentrations in three Japanese patients with PR were measured. We also cloned PYCARD/ASC cDNA variants and expressed them in THP-1 cells to determine their effects on inflammasome activity following stimulation with phorbol 12-myristate 13-acetate and monosodium urate. Lysates of recombinant THP-1 cells were subjected to co-immunoprecipitation assays.

Results: Serum IL-1 $\beta$ concentrations were significantly elevated in patients with PR, and a splice variant of PYCARD/ ASC mRNA lacking exon 2 ( $\Delta$ exon2) was dominantly expressed compared with that in controls. Moreover, IL- $1 \beta$ secretion was significantly increased in THP-1 cells expressing $\triangle$ exon2 PYCARD/ASC compared with that in cells expressing the wild-type protein. The amount of NLRP3 bound to $\triangle$ exon2 PYCARD/ASC was increased after stimulation, whereas that bound to the wild-type protein was decreased. There were no differences in caspase-1 binding.

Conclusion: $\triangle$ exon2 PYCARD/ASC was associated with the pathogenesis of PR.

Key words: palindromic rheumatism, inflammasome, PYD and CARD domain-containing protein/apoptosis-associated speck-like protein containing a CARD, splice variant, interleukin-1 $\beta$

\section{From:}

${ }^{1}$ Division of Medical Biophysics, Department of Biophysics,

Graduate School of Health Sciences, Kobe University, Kobe, Japan

${ }^{2}$ Shichikawa Arthritis Research Center, Osaka Rehabilitation Hospital, Hannan, Japan

Institute for Rheumatic Diseases, Nagahama, Japan

\author{
* Corresponding author: \\ Koichiro Komai \\ Division of Medical Biophysics, Department of Biophysics, \\ Graduate School of Health Sciences, Kobe University \\ 7-10-2, Tomogaoka Suma, Kobe 654-0142, Hyogo, Japan \\ E-mail: komai@kobe-u.ac.jp
}

\section{Introduction}

Palindromic rheumatism (PR) is a rare periodicity arthritis that is characterized by relapsing short episodes of arthritis. ${ }^{1}$ Although the pathogenesis of PR is unclear, the clinical features of PR are similar to those of auto-inflammatory diseases. ${ }^{2}$ Auto-inflammatory diseases are clinically characterized by recurrent episodes of fever, systemic inflammation, and symptoms such as skin rashes, abdominal pain, chest pain, lymphadenopathy, and arthritis, without high-titer autoantibodies or antigen-specific T lymphocytes. These diseases are caused by dysregulation of the innate immune system. ${ }^{3}$ Innate immune function depends on pattern-recognition receptors (PRRs), which recognize pathogen-associated molecular patterns (PAMPs) derived from invading pathogens, and danger-associated molecular patterns (DAMPs) induced as a result of endogenous stress. One representative PRR is the NLR family, including NLRP3. NLRs are signal transduction molecules 
that modulate inflammatory signaling by forming inflammasomes. $^{4}$

Inflammasomes are multimeric protein complexes localized in the cytoplasm; these complexes play important roles in inflammation and induce cytokine secretion, including secretion of interleukin (IL), $-1 \beta$ and IL-18, via activation of caspase-1. A typical inflammasome consists of PRRs, PYD and CARD domain containing/apoptosis-associated speck-like protein containing a CARD (PYCARD/ASC), and caspase-1. Therefore, auto-inflammatory diseases are thought to be caused by primary dysregulation of inflammasomes owing to abnormalities in these inflammasome-related genes. ${ }^{5}$

Based on similarities between the pathological features of PR and autoinflammatory diseases, dysregulation of inflammasome components is suspected. ${ }^{2}$ Although mutations in inflammasome-related genes, such as $M E F V$, have previously been reported in patients with $\mathrm{PR}$, no reports have described an association between $\mathrm{PR}$ and the PYCARD/ASC gene, which encodes a common inflammasome adapter protein. ${ }^{6,7}$ Accordingly, in this study, we analyzed the status and function of PYCARD/ASC in Japanese patients with PR.

\section{Materials and Methods \\ Patients}

We included three Japanese women with PR (designated as PR1-3) in this study. All patients had been examined at Shichikawa Arthritis Center, Osaka Rehabilitation Hospital. The study was approved by the institutional ethical committee of Kobe University Graduate School of Health Sciences and was conducted according to the principles of the Declaration of Helsinki (approval no. 140-3).

PR1 was a 42-year-old Japanese woman. Blood tests revealed the following parameters: C-reactive protein (CRP), 0.34 $\mathrm{mg} / \mathrm{dL}$; erythrocyte sedimentation rate (ESR), $11 \mathrm{~mm} / \mathrm{h}$; matrix metalloproteinase (MMP)-3, $43.9 \mathrm{ng} / \mathrm{mL}$; uric acid (UA), $3.2 \mathrm{mg} / \mathrm{dL}$; rheumatoid factor (RAHA), < 40 times; anti-cyclic citrullinated peptide (CCP) antibody, $1.0 \mathrm{U} / \mathrm{mL}$ (negative); anti-DNA antibody, negative; antinuclear antibodies, 40×; and lupus erythematosus (LE) test, negative.

PR2 and PR3 were familial. PR2 was a 60-year-old Japanese woman who experienced the onset of PR at 57 years of age. PR2 was the mother of PR3. Blood tests revealed the following parameters for PR2: CRP, $2.35 \mathrm{mg} / \mathrm{dL}$; ESR, $53 \mathrm{~mm} / \mathrm{h}$; MMP-3, 19.0 ng/mL; RAHA, 40×; anti-CCP antibody, $0.6 \mathrm{U} /$ $\mathrm{mL}$ (negative); antinuclear antibodies, $40 \times$. PR3 was 27 years old, and PR onset occurred at 22 years of age. Blood tests revealed the following parameters: CRP, $2.96 \mathrm{mg} / \mathrm{dL}$; ESR, $21 \mathrm{~mm} / \mathrm{h}$; MMP-3, $19.5 \mathrm{ng} / \mathrm{mL}$; UA, $2.9 \mathrm{mg} / \mathrm{dL}$; RAHA, 40×; anti-CCP antibody, $2.4 \mathrm{U} / \mathrm{mL}$ (negative); antinuclear antibodies, $40 \times$. Serum IL-1 $\beta$ concentrations were measured using a human IL- $1 \beta$ enzyme-linked immunosorbent assay (ELISA) kit (Diaclone, Besancon, France).

\section{RNA isolation and reverse transcription polymerase chain re- action (RT-PCR)}

Total RNA from peripheral blood samples was isolated using a PAXgene blood RNA kit (Qiagen, Hilden, Germany) and then reverse transcribed into cDNA with $0.5 \mu \mathrm{M}$ oligo $\mathrm{dT}$ primer in $20 \mu \mathrm{L}$ PCR buffer II containing $2.5 \mathrm{mM} \mathrm{MgCl}_{2}, 0.4$ mM dNTP mixture, $1 \mathrm{U}$ RNase inhibitor, and $2.5 \mathrm{U}$ MuLV reverse transcriptase (Life Technologies, CA, USA) at $25^{\circ} \mathrm{C}$ for $10 \mathrm{~min}, 42^{\circ} \mathrm{C}$ for $20 \mathrm{~min}$, and $99^{\circ} \mathrm{C}$ for $5 \mathrm{~min}$. PYCARD/ ASC cDNA was amplified by PCR in tubes containing $0.2 \mathrm{mM}$ each dNTP, $1 \times$ Ex Taq Buffer (Takara Bio, Shiga, Japan), 0.2 $\mu \mathrm{M}$ each primer, and $1.25 \mathrm{U}$ Ex Taq DNA polymerase (Takara Bio) for 30 cycles at $94^{\circ} \mathrm{C}$ for $30 \mathrm{~s}, 55^{\circ} \mathrm{C}$ for $30 \mathrm{~s}$, and $72^{\circ} \mathrm{C}$ for $2 \mathrm{~min}$, followed by a final extension at $72^{\circ} \mathrm{C}$ for $5 \mathrm{~min}$. Primers for amplification were as follows: $h A S C 139 F$ forward, $5^{\prime}$ AGGAGCTCAAGAAGTTCAAG-3'; hASC729R reverse, 5'AGGATGATTTGGTGGGAT-3'.

\section{Transient expression of PYCARD/ASC}

To construct wild-type PYCARD/ASC and $\triangle$ exon2 PYCARD /ASC expression vectors, wild-type and $\triangle$ exon2 PYCARD/ASC fragments were amplified by PCR using PYCARD/ASC-specific primers (forward primer, 5'-CTCAAGCTTCGAATTCCGACT TCCTCCTGGTCGG-3'; reverse primer, 5'-GTCGACTGCAG AATTCGGAGTGTTGCTGGGAAGG-3'). PCR products were cloned into an EcoRI-digested pcDNA3.1(+) vector (Thermo Fisher Scientific, MA, USA) using an In-Fusion cloning kit (Takara Bio).

THP-1 cells purchased from JCRB Cell Bank were plated at a density of $5.0 \times 10^{5}$ to $1.5 \times 10^{6}$ cells $/ \mathrm{mL}$ in RPMI1640 medium supplemented with $100 \mathrm{mU} / \mathrm{mL}$ penicillin (Thermo Fischer Scientific), $100 \mu \mathrm{g} / \mathrm{mL}$ streptomycin (Thermo Fischer Scientific), and 10\% fetal bovine serum (Biowest, FL, USA) and incubated overnight. Transfection experiments were performed using TransIT-LT1 reagent (Mirus Bio, WI, USA) according to the manufacturer's instructions. Next, $500 \mu \mathrm{L}$ Opti-MEM I reduced serum medium (Thermo Fisher Scientific), $15 \mu \mathrm{L}$ TransIT-LT1 reagent, and $1 \mu \mathrm{g}$ pcDNA3.1/wild-type PYCARD/ASC or pcDNA3.1/ $\triangle$ exon2 PYCARD/ASC were added to THP-1 cells and incubated for $48 \mathrm{~h}$. Total RNA from THP-1 cells transfected with recombinant vectors was then isolated using RNAiso (Takara Bio), and cDNAs were reverse transcribed by using a PrimeScript II $1^{\text {st }}$ Strand cDNA Synthesis Kit (Takara Bio). PYCARD/ASC mRNA expression was confirmed by RT-PCR using the following primer pairs: forward primer ( $h P Y C A R D$ 225F), 5'-ATGGACGCCTTGGACCTCA-3'; reverse primer (hPYCARD696R), 5'-GGAGTGTTGCTGGGAAGGA-3'. RTPCR was performed using AmpliTaq Gold DNA polymerase (Thermo Fischer Scientific) at $95^{\circ} \mathrm{C}$ for $12 \mathrm{~min}$; 35 cycles of $94^{\circ} \mathrm{C}$ for $30 \mathrm{~s}, 60^{\circ} \mathrm{C}$ for $30 \mathrm{~s}$, and $72^{\circ} \mathrm{C}$ for $2 \mathrm{~min}$; and $72^{\circ} \mathrm{C}$ for $5 \mathrm{~min}$.

\section{Inflammasome activation assay}

Recombinant THP- 1 cells at a density of $1.5 \times 10^{6}$ cells $/ \mathrm{mL}$ were pretreated with $0.5 \mu \mathrm{M}$ phorbol-12-myristate-13-acetate (PMA; Wako Pure Chemical Industries, Tokyo, Japan) for $3 \mathrm{~h}$. After washing with RPMI1640, cells were plated at a density of $3.0 \times 10^{5}$ cells $/ \mathrm{mL}$ and incubated overnight. Cells were then plated at a density of $1.2 \times 10^{6}$ cells $/ \mathrm{mL}$ in $500 \mu \mathrm{L}$ serum-free medium and stimulated with $100 \mu \mathrm{g} / \mathrm{mL}$ monosodium urate (MSU; AdipoGen, CA, USA) for $6 \mathrm{~h}$. Total IL-1 $\beta$ concentrations in conditioned medium were measured using a human IL-1 $\beta$ ELISA kit (Diaclone). 


\section{Co-immunoprecipitation}

Recombinant THP-1 cells were lysed in CelLytic M reagent (Sigma-Aldrich, MO, USA) containing protease inhibitor cocktail (Roche, Basel, Switzerland). Protein coupled with the anti-ASC antibody (cat. no. B-3; Santa Cruz Biotechnology, Dallas, TX, USA) was obtained using a Dynabeads Protein G Immunoprecipitation Kit (Thermo Fischer Scientific) according to the manufacturer's protocol. Lysates and immunoprecipitated samples were then mixed with $5 \times$ electrophoresis sample buffer and subjected to sodium dodecyl sulfate-polyacrylamide gel electrophoresis using 10-20\% polyacrylamide gradient gels (Biocraft, Tokyo, Japan). Electroblotted transfer membranes were blocked with $5.0 \%$ skim milk in phosphate-buffered saline containing $0.05 \%$ Tween-20 and then reacted with primary antibodies at $4^{\circ} \mathrm{C}$ overnight. After washing, the membranes were incubated at $4^{\circ} \mathrm{C}$ for overnight. The proteins were detected using Amersham ECL Prime western blotting detection reagent (GE Healthcare, Buckinghamshire, UK). The following antibodies were used for detection of ASC, caspase-1, and NLRP3: anti-ASC antibodies (cat. no. B-3; Santa Cruz Biotechnology), anti-NLRP3 antibodies (cat. no. D4D8T; Cell Signaling Technology, Danvers, MA, USA), and anti-caspase-1 antibodies (cat. no. D7F10; Cell Signaling Technology). VeriBlot was used for the secondary antibody for immunoprecipitation (conjugated to horseradish peroxidase; cat. no. ab131366; Abcam, Cambridge, UK), and Amersham ECL Anti-Mouse IgG, Horseradish Peroxidase-linked species-specific whole antibody (GE Healthcare) was used as another secondary antibody.

\section{Statistical analysis}

All statistical analyses were performed using two-sample Welch's t-tests or Student's t-tests. Differences with a $P$ value of less than 0.05 were considered significant.

\section{Results}

Serum IL-1 $\beta$ concentrations were significantly elevated in patients with $P R$

Serum IL- $1 \beta$ concentrations in patients with PR $(n=3$; $27.6 \pm 15.1 \mathrm{pg} / \mathrm{mL}$ [mean \pm standard error]) were significantly higher than those in controls $(\mathrm{n}=7 ; 6.8 \pm 1.7 \mathrm{pg} / \mathrm{mL} ; P<$ 0.05 ; Figure 1). Thus, these findings suggested that the inflammasome was activated in patients with PR.

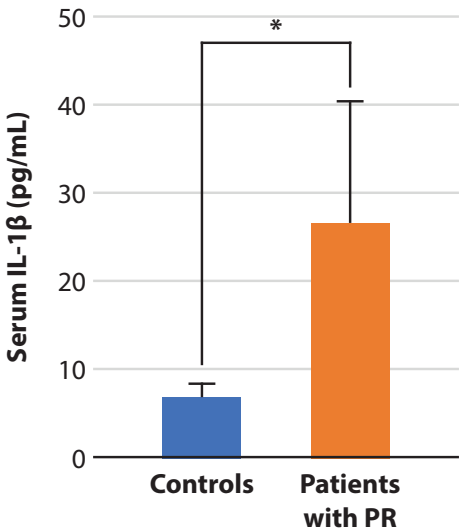

Figure 1. Serum IL-1 $\beta$ concentrations in controls $(n=7)$ and patients with $\mathrm{PR}(\mathrm{n}=3) .{ }^{\star} P<0.05$.

The PYCARD/ASC splice variant lacking exon 2 (Aexon2) was dominantly expressed in patients with $P R$

We next isolated PYCARD/ASC cDNA from patients with $\mathrm{PR}$ and found that exon 2 was dominantly absent in all patients with PR compared with controls (Figure 2a, b). We also confirmed that exon 2 was conserved in genomic DNA in all patients with PR (Figure 2c). Therefore, these findings indicated that exon 2 in the mRNA was lost during RNA splicing in all patients with PR.

\section{IL-1 $\beta$ secretion was significantly increased in THP-1 cells ex- pressing $\Delta$ exon 2 PYCARD/ASC}

To investigate whether $\triangle$ exon2 PYCARD/ASC affected inflammasome function, we generated THP-1 cells expressing recombinant wild-type or $\triangle$ exon2 PYCARD/ASC and measured IL-1 $\beta$ secretion following stimulation with PMA or PMA plus MSU. The results showed that IL- $1 \beta$ secretion following PMA stimulation was significantly increased in cells expressing $\Delta \mathrm{ex}^{-}$ on2 PYCARD/ASC $(43.4 \pm 2.2 \mathrm{pg} / \mathrm{mL})$ compared with that in cells expressing the wild-type protein $(24.8 \pm 3.8 \mathrm{pg} / \mathrm{mL} ; P<$ 0.05 ; Figure 3a, b). Although IL- $1 \beta$ secretion was also increased in cells expressing $\Delta$ exon2 PYCARD/ASC after PMA plus MSU stimulation, this difference was not significant. (a)

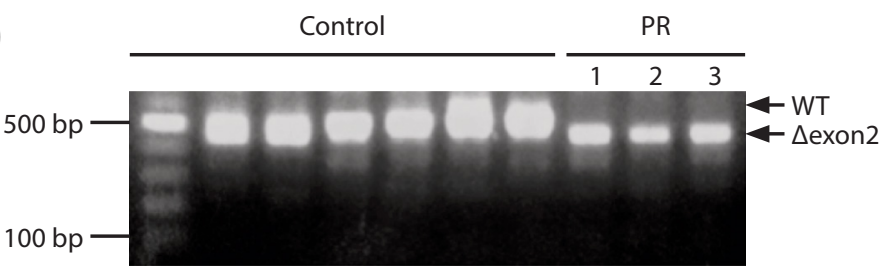

(b)
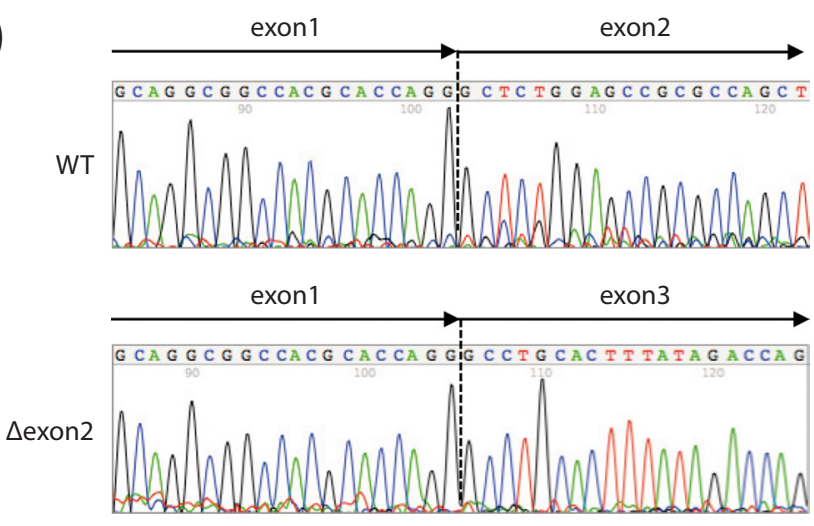

Figure 2. A splice variant of PYCARD/ASC cDNA lacking exon 2 ( $\triangle$ exon2) was found in patients with PR. (a) Expression of $P Y C A R D / A S C$ in controls and patients with PR. (b) Wild-type and $\triangle$ exon $2 P Y C A R D / A S C$ cDNA sequences. 
(c)

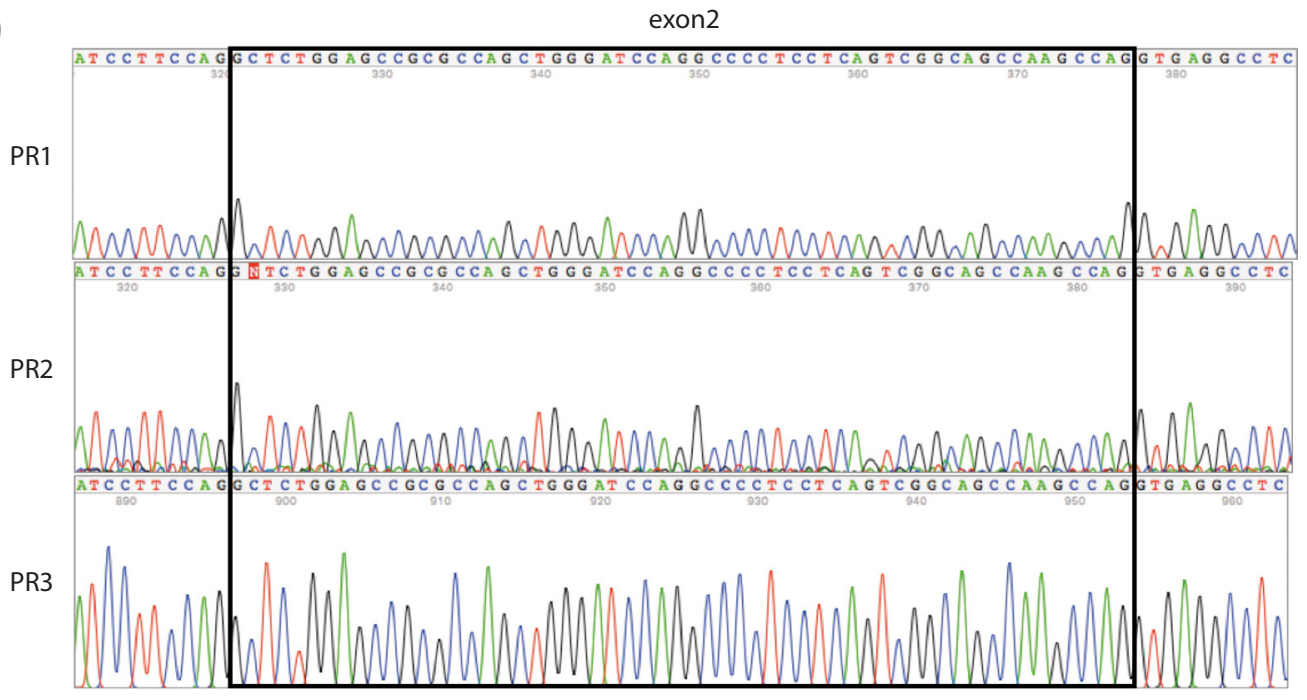

Figure 2. (Continued) (c) Genomic sequences of PYCARD/ASC exon 2 in PR1-3.

(a)

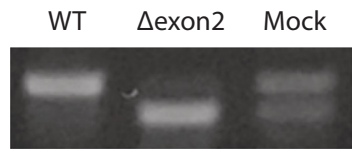

(b)

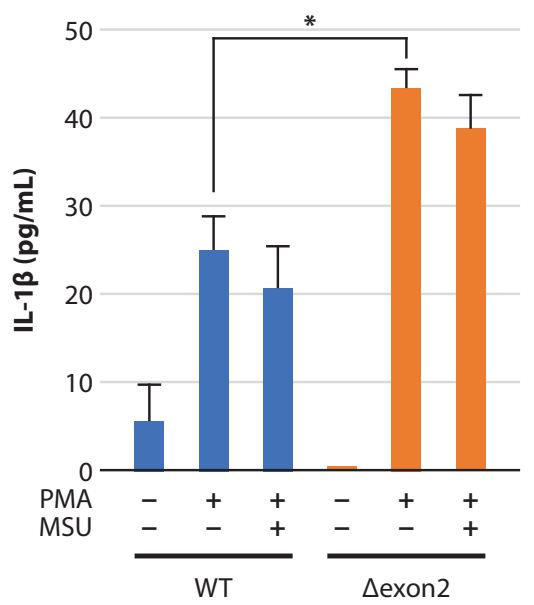

(c)

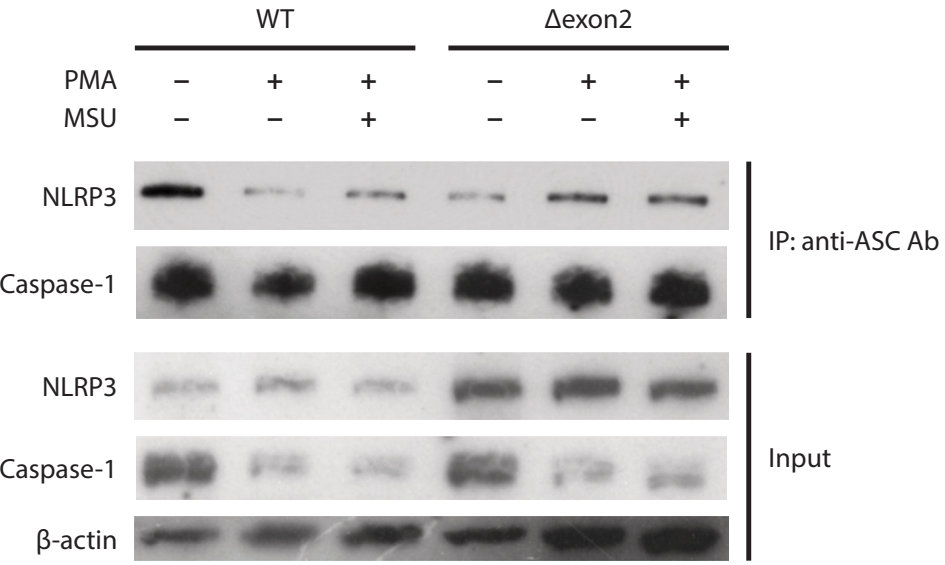

Figure 3. Functional analysis of $\triangle$ exon2 PYCARD/ASC. (a) Expression of recombinant PYCARD/ASC in THP-1 cells was confirmed using RT-PCR. (b) IL-1 $\beta$ concentrations in the medium of THP-1 cells expressing the recombinant protein after stimulation with PMA and MSU. ${ }^{\star} P<0.05$. (c) Western blotting of immunoprecipitated fractions using anti-PYCARD/ASC antibodies and cell lysates from recombinant THP-1 cells, detected using anti-NLRP3 and anti-caspase-1 antibodies.

Because abnormalities in the NLRP3 inflammasome cause several auto-inflammatory diseases, we performed co-immunoprecipitation to investigate the binding of PYCARD/ASC variants with NLRP3 or caspase-1. The amount of NLRP3 bound with PYCARD/ASC was increased by stimulation with PMA or PMA plus MSU in THP-1 cells expressing $\triangle$ exon2 PYCARD/ASC but was decreased in cells expressing wild-type PYCARD/ASC. There were no changes in caspase-1 binding (Figure 3c).

\section{Discussion}

In this study, we found that $\triangle$ exon2 PYCARD/ASC was expressed in patients with $\mathrm{PR}$ and promoted inflammasome activation compared with the wild-type protein. Although this variant has been found in HL-60 and THP-1 cells, ${ }^{8,9}$ this is the first identification of this PYCARD/ASC splice variant in patients with PR.

The mechanisms mediating the expression levels of $\Delta$ exon2 PYCARD/ASC are still unclear. Because inflammasome -associated gene mutations have been found in patients with several auto-inflammatory diseases, ${ }^{10}$ it is possible that genetic mutations affect PYCARD/ASC splicing in patients with $\mathrm{PR}$. The priming signal for inflammasome activation involves Toll-like receptor-4 (TLR-4), tumor necrosis factor receptor 1, and IL-1R. ${ }^{11}$ Additionally, Bryan et al. reported that $\Delta$ exon2 PYCARD/ASC expression is increased by lipopolysaccharide, 
a ligand for TLR-4. ${ }^{9}$ Consistent with this, we found that serum IL- $1 \beta$ concentrations were significantly higher in patients with PR than in controls, suggesting that the priming signal pathway involving IL-1R may be activated in patients with PR and that this may result in an increase in $\triangle$ exon2 PYCARD/ASC expression. Thus, further studies are needed to elucidate the mechanisms mediating the expression of $\triangle$ exon2 PYCARD/ ASC.

PYCARD/ASC functions as a mediator of the inflammatory signaling pathway as a downstream effector of TLR signaling. PYCARD/ASC has been shown to activate caspase-1 through its oligomerization in inflammasomes and to process pro-IL-1 $\beta$ and pro-IL-18 into mature IL- $1 \beta$ and IL-18, respectively, leading to the establishment of innate immunity. Matsushita et al. showed that higher levels of IL-1 $\beta$ are secreted in COS-7 cells expressing $\triangle$ exon2 PYCARD/ASC compared with that in cells expressing wild-type protein. ${ }^{8}$ In contrast, Bryan et al. reported that $\triangle$ exon2 PYCARD/ASC reduces IL- $1 \beta$ secretion in THP- 1 cells. ${ }^{9}$ Thus, we measured IL- $1 \beta$ secretion from recombinant THP-1 cells expressing wild-type and $\triangle$ exon2 PYCARD/ASC. The results showed that PMAinduced IL- $1 \beta$ secretion was significantly higher in cells expressing $\triangle$ exon2 PYCARD/ASC than in cells expressing wildtype protein. Exon 2 encodes the PGR domain between PYD and CARD and may mediate the flexibility of PYCARD/ASC, helping to create a hinge between PYD and CARD that is folded in the inactive form of PYCARD/ASC. ${ }^{8}$ Therefore, as previously described by Matsushita et al., $\Delta$ exon2 PYCARD/ASC protein is thought to be inflexible and may be constitutively activated. Therefore, although the binding capacity of $\Delta$ exon 2 PYCARD/ASC with caspase-1 was unaffected, as compared with that of the wild-type protein, we hypothesize that $\Delta \mathrm{ex}^{-}$ on 2 ASC may lead to excess IL- $1 \beta$ secretion. Moreover, we also found that the amount of NLRP3 bound with PYCARD/ASC was increased by stimulation in cells expressing $\Delta$ exon2 PYCARD/ASC but was decreased in cells expressing wild-type protein. Therefore, the amount of stable-inflammasome complex, comprised of NLRP3, $\triangle$ exon2 PYCARD/ASC, and caspase-1, may be increased compared with that of wildtype PYCARD/ASC and may affect inflammasome activity. Interestingly, although NLRP3 interacted with PYCARD/ASC without stimulation in THP-1 cells expressing recombinant wild-type protein, IL- $1 \beta$ secretion was lower than that in stimulated cells. Because recruitment of NLRP3 to phagophores is required for interaction with $\mathrm{PYCARD/ASC},{ }^{12}$ our results suggested that wild-type PYCARD/ASC expression may enhance NLRP3 autophagic degradation by promoting the interactions between NLRP3 and PYCARD/ASC in an unstimulated state. Additionally, NLRP3 protein expression levels in peripheral blood mononuclear cells of patients with PR were elevated compared with that in controls (data not shown). Thus, NLRP3 inflammasome activity may be enhanced and may participate in the pathogenesis of PR.

Recent studies have reported that inflammatory diseases, as well as other conditions (e.g., gout, Alzheimer's disease, and others), are affected by abnormalities in inflammasomes. ${ }^{13}$ Thus, further studies are required to analyze the functions and the splicing mechanism of the PYCARD/ASC gene to determine its role in other disease states.
In conclusion, we found that serum IL- $1 \beta$ concentrations were elevated in patients with PR and that $\triangle$ exon2 PYCARD/ ASC mRNA was predominantly expressed in comparison with controls. We also found that $\triangle$ exon2 PYCARD/ASC is involved in dysregulation of inflammation, suggesting that it plays a critical role in the pathogenesis of PR. Therefore, the $\Delta$ exon2 PYCARD/ASC variant may be a target for the diagnosis and treatment of patients with PR.

\section{Acknowledgements}

We would like to thank Editage (www.editage.jp) for English language editing.

\section{Conflicts of Interest None.}

\section{Source of Funding}

This work was supported by Ito Chubei Foundation, General Incorporated Foundation, Japan, and JSPS KAKENHI (grant no. 16K08934).

\section{Author Contributions}

- Yumi Suganuma, Aya Kawase, Aoi Kishida, Moeko Yamaguchi, and Atsuko Yabuuchi carried out functional analysis.

- Hayate Tanaka found PYCARD/ASC splice variant in patients with PR.

- Koji Inoue diagnosed patients with PR.

- Shunichi Shiozawa designed the research.

- Koichiro Komai designed all experiments, wrote grants, and wrote and edited the manuscript.

\section{References}

1. Sanmarti R, Cañete JD, Salvador G. Palindromic rheumatism and other relapsing arthritis. Best Pract Res Clin Rheumatol. 2004;18:647-61.

2. Ben-Chetrit E, Peleg H, Aamar S, Heyman SN. The spectrum of MEFV clinical presentations--is it familial Mediterranean fever only? Rheumatology (Oxford). 2009;48:1455-9.

3. Wekell P, Karlsson A, Berg S, Fasth A. Review of autoinflammatory diseases, with a special focus on periodic fever, aphthous stomatitis, pharyngitis and cervical adenitis syndrome. Acta Paediatr. 2016;105: 1140-51.

4. Guo H, Callaway JB, Ting JP. Inflammasomes: mechanism of action, role in disease, and therapeutics. Nat Med. 2015;21:677-87.

5. Touitou I, Koné-Paut I. Autoinflammatory diseases. Best Pract Res Clin Rheumatol. 2008;22:811-29.

6. Cañete JD, Arostegui JI, Queiró R, Gratacós J, Hernández MV, Larrosa $\mathrm{M}$, et al. An unexpectedly high frequency of MEFV mutations in patients with anti-citrullinated protein antibody-negative palindromic rheumatism. Arthritis Rheum. 2007;56:2784-8.

7. Masumoto J, Taniguchi S, Ayukawa K, Sarvotham H, Kishino T, Niikawa N, et al. ASC, a novel 22-kDa protein, aggregates during apoptosis of human promyelocytic leukemia HL-60 cells. J Biol Chem. 1999;274(48):33835-8.

8. Matsushita K, Takeoka M, Sagara J, Itano N, Kurose Y, Nakamura A, et al. A splice variant of ASC regulates IL-1 release and aggregates differently from intact ASC. Mediators Inflamm. 2009;2009:287387.

9. Bryan NB, Dorfleutner A, Kramer SJ, Yun C, Rojanasakul Y, Stehlik C. Differential splicing of the apoptosis-associated speck like protein containing a caspase recruitment domain (ASC) regulates inflammasomes. J Inflamm (Lond). 2010;7:23.

10. Ozen S. What's new in autoinflammation?. Pediatr Nephrol. [Preprint]. 2018 [cited 2019 Feb 1]:[8 p.]. Available from: https://link.springer.com/ article/10.1007\%2Fs00467-018-4155-4 
11. Verstrepen L, Bekaert T, Chau TL, Tavernier J, Chariot A, Beyaert R. TLR-4, IL-1R and TNF-R signaling to NF-kB: variations on a common theme. Cell Mol Life Sci. 2008;65:2964-78.

12. Spalinger MR, Lang S, Gottier C, Dai X, Rawlings DJ, Chan AC, et al. PTPN22 regulates NLRP3-mediated IL1B secretion in an autophagy -dependent manner. Autophagy. 2017;13(9):1590-601.
13. Mitroulis I, Skendros P, Ritis K. Targeting IL-1beta in disease; the expanding role of NLRP3 inflammasome. Eur J Intern Med. 2010;21: 157-63. 